\title{
Enhancing Pool Boiling Heat Transfer by Structured Surfaces-A Lattice Boltzmann Study
}

\author{
Y. Huang ${ }^{1}$, Y. Tian ${ }^{2}$, W. Ye ${ }^{1}$, W. Li ${ }^{1}$, J. Lei ${ }^{1}$ and Y. Zhang ${ }^{1 \dagger}$ \\ ${ }^{1}$ School of Mechatronics Engineering, Nanchang University, Nanchang, Jiangxi, 330031, China \\ ${ }^{2}$ Institute of Energy and Sustainable Development (IESD), School of Engineering and Sustainable \\ Development, De Montfort University, Leicester LE1 9BH, England, UK \\ †Corresponding Author Email: yzhan2033@163.com
}

(Received February 18, 2021; accepted August 17, 2021)

\begin{abstract}
The structured surface-enhanced pool boiling process and associated heat transfer enhancement characteristics are numerically investigated by using the pseudopotential multiphase flow lattice Boltzmann (LB) model coupled finite difference method (FDM). In the current study, the effects of different microstructure geometries(square structures, triangular serrated structures, triangular-raised structures) and varying spacing in triangular-raised structures $(d=0,22,44,66,88,110$ l.u. (lattice units)) on boiling heat transfer (BHT) characteristics and bubble dynamics behavior are studied in detail. The results showed that microstructure can accelerate bubble nucleation. Among the three microstructures, the heat transfer performance of triangularraised structures was significantly better than that of square and triangular serrated structures in the nucleate boiling ( $\mathrm{Ja}$ number is $0.124-0.145)$. The oscillation and deformation of bubbles led to the lateral migration of bubbles, the continuous nucleation of small bubbles. The phenomenon of re-wetting of heating surface occurred in the process of bubble migration, necking, deformation and detachment was found, which enhances heat transfer in nucleate boiling. At the same time, the growth, oscillation and detachment of bubbles also perturb the liquid and enhance the natural convection around the bubbles.Therefore the main BHT mechanism of nucleate boiling on the three kinds of structured surface is the combined action of transient heat conduction and micro-convection. The variation of the spacing between microstructures showed an important effect on the BHT performance of the heating surface and the generation of activated nucleation sites in the nucleate boiling. Triangular-raised structures can enhance transient heat conduction and micro-convection, with the strongest enhancement effect at $d=661$.u when the $J a$ number is 0.124 .
\end{abstract}

Keywords: Boiling; Structured surface; Bubble dynamics; Lattice Boltzmann method.

\section{NOMENCLATURE}

a parameter in P-R state equation a parameter in P-R state equation

lattice speed

lattice sound speed of the D2Q9 model specific heat at constant volume distance between microstructure initial droplet diameter stable droplet diameter droplet detachment diameter

discrete velocity in the $i$ direction density distribution function density equilibrium distribution function total force fluid-solid pseudopotential interaction force forcing term within the velocity space buoyancy force
$T_{\mathrm{c}} \quad$ critical temperature

$T_{h} \quad$ temperature of the center three grids on the bottom wall

$T_{\mathrm{s}} \quad$ saturation temperature

$\boldsymbol{u}$ macroscopic velocity

$u_{x} \quad$ velocity in the $\mathrm{x}$ direction

$u_{y} \quad$ velocity in the $y$ direction

$w_{i} \quad$ weights

$x \quad$ spatial position

$\left(x_{0}, y_{0}\right) \quad$ central coordinate of the computing domain

\section{Greek symbols}

$\delta_{t} \quad$ time step

$\Delta T \quad$ superheat on the heating surface

$\theta$ equilibrium contact angles at the saturation temperature $\begin{array}{ll}\kappa & \text { acentric factor } \\ \rho & \text { macroscopic density }\end{array}$ 


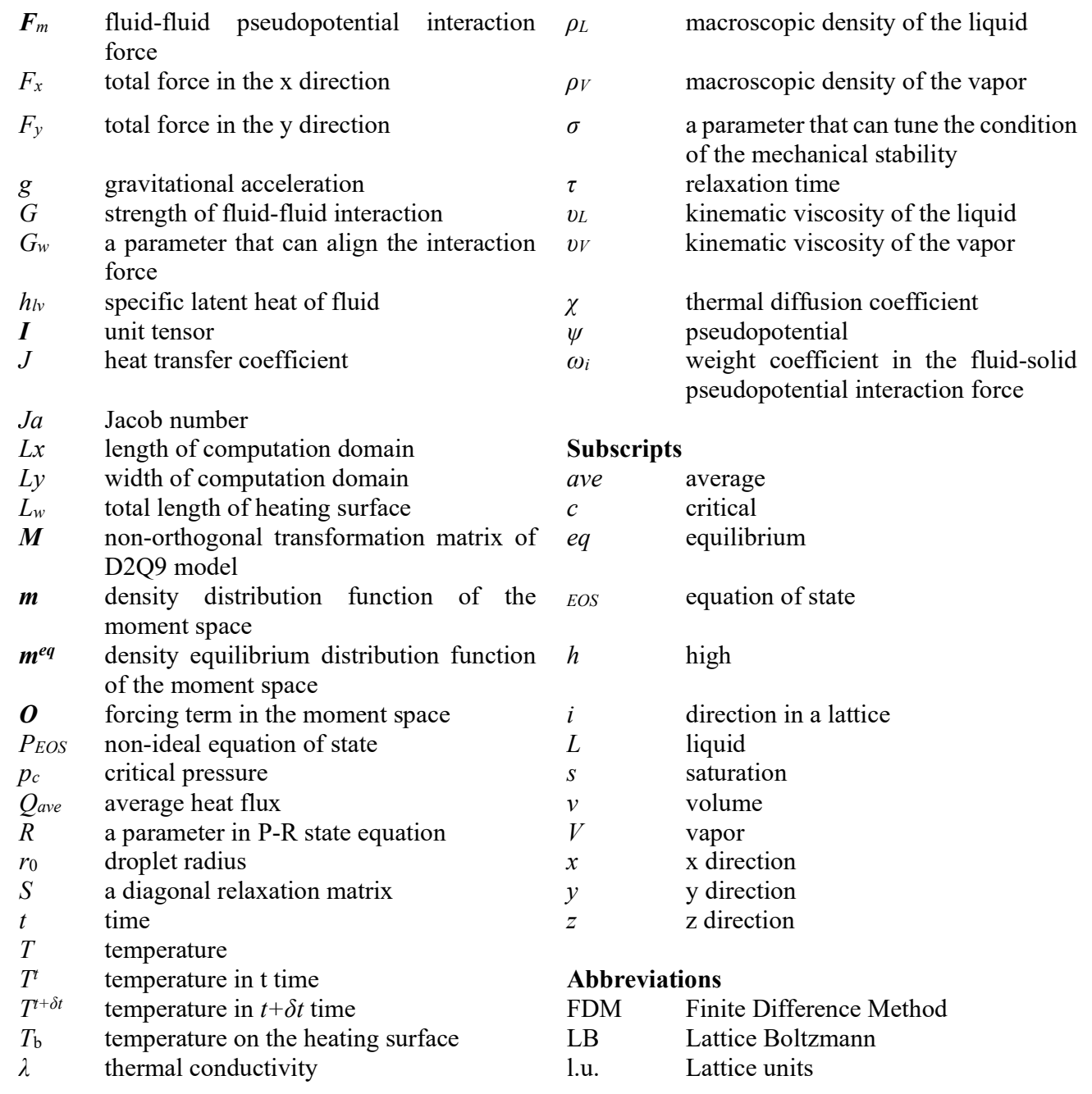

\section{INTRODUCTION}

Nucleate boiling is an efficient way to transfer heat, which takes away significant latent heat through the generated bubbles, thus dissipating large heat fluxes. Because of its excellent heat dissipation capacity, it is widely used in engineering and industrial fields, such as nuclear reactors, power plants, electronic equipment cooling, refrigeration and chemical process (Dhir et al. 2013; Hamzekhani et al. 2015). In the early studies of boiling, researchers have conducted many experiments to study the boiling heat transfer (BHT) mechanism, the boiling phenomena, the boiling curve and so on, with some significant progress made (Wei et al. 2005; Chu et al. 2012). These developments have deepened our understanding of the boiling process and solved some problems in engineering and industrial fields. Thereupon many technologies were proposed to enhance the heat transfer performance of nucleate boiling. Among them, the surface modification technology has been widely used due to its high potential to enhance the heat transfer performance by providing larger surface area, higher nucleation site density and potentially smaller phase transition overheating (Thiagarajan et al. 2015). Surface

modification technology has been commonly adopted in two methods: one is to control the wettability of the boiling surface (Jo et al. 2011), and the other is to implement micro-nano machining on the boiling surface (Kim et al. 2015a).

The physical mechanism of boiling on structured surface is complex, which involves the dynamics of interaction between vapor, liquid, the solid heating surface and a series of complex phenomena such as flow field disturbance. Therefore, many experiments have been conducted to study the BHT mechanism and the physical phenomena in the boiling process (Xie et al. 2021a, 2021b, 2020; Zonouzi et al. 2018, Halon et al. 2018; Kim et al. 2015; Tang et al. 2016). Xie et al. (2021a) designed experiments to investigate the pool boiling heat transfer characteristics of multi-oriented hierarchical structured surfaces. The results showed that nanostructured surfaces exhibit better boiling performance than all multi-oriented planar surfaces. The departure time and fully developed vapor film thickness of the inclined surfaces increased with increasing surface orientation. Halon et al. (2018) designed two different kinds of enhanced boiling surfaces: NTS (narrow tunnel structure) and TS (tunnel structure), and studied the low-pressure 
boiling phenomenon and BHT performance of these two structured surfaces. Their results indicated that the BHT performance of tunnel structure was significantly improved at lower pressure, at even one order of magnitude higher than that of a flat surface. Kim et al. (2015b) performed an experimental study on the effect of structured surface on BHT characteristics. Their results revealed that with the increase of surface roughness, the BHT performance increased but the enhancement slowed down gradually. Tang et al. (2016) designed a porous interconnected micro-channel network structured surface, and carried out a BHT performance research on the prepared surface. The results showed that the channel width had a significant effect on the BHT coefficient.

In boiling research, it is not sufficient enough to just rely on the experimental method because the experimental results are often contradictory due to complexity, many factors affecting the boiling curve are indistinguishable in the experimental study (Jo et al. 2011; O'hanley et al. 2013). For the boiling of structured surfaces, there are certain limitations in the experimental research, such as the experimental cost, the manufacturing accuracy of structured surface, the accurate observation of bubble motion (Dong et al. 2020), etc. Therefore, numerical simulation has become an important means to investigate the potential BHT mechanism and characteristics involved in the boiling process on structured surfaces. The Lattice Boltzmann (LB) method is a mesoscopic method based on kinetic equations, and in recent year has received extensive attention in simulating complex fluid systems such as two-phase flow and vapor-liquid phase transition, having shown great potential (Dong et al. 2020; Zhao et al. 2021, 2019a, 2019b; Chang et al. 2019; Zhang et al. 2020; Zhou et al. 2019; Bi et al. 2019). Dong et al. (2020) performed research on the BHT characteristics and bubble dynamics behavior during subcooled boiling on an inclined surface using lattice Boltzmann method. The effects of inclination angle and surface geometry on bubble motion and BHT performance were discussed in detail. The results showed that the BHT coefficient was not only related to the inclination angle, but also to the surface geometry. Zhao et al. (2021) studied the effect of confined space and free outlet boundary on the heat transfer characteristics of nucleation boiling under conjugate heat transfer using phase change lattice Boltzmann method. The obvious sliding growth phenomenon and curved rising trajectory and large deformation of the rising vapor bubble were observed in their study. Chang et al. (2019) used the thermal two-phase LB model to numerically simulate the enhanced BHT characteristics on the columnar structured surface. The effects of column height, column width and spacing on the BHT performance were analyzed in detail. The results revealed that the BHT performance mainly depends on the heating area and the local convection flow field. Zhou et al. (2019) used the LB model with two-particle distribution functions to study the bubble dynamic behavior during the boiling process of micro-pillar structured surface, including the periodic nucleation, growth, and detachment process of bubbles, and analyzed in detail the effects of micro-pillar spacing and the height on bubble growth and detachment. The results showed that with the decrease of micropillar height, the heat flux increased and the departure period of nucleated bubbles shortened, while the spacing between two micro-pillars showed no distinct effect despite having great effect on the formation of activated nucleation sites. Although the LBM method has its own limitations that can not be well compared with the conditions used in experimental studies, from the above researchers using the LBM method and the conclusions drawn in the process of simulating boiling, the method can obtain the fine and detailed characterization of the bubble dynamics processes such as bubble nucleation, necking, deformation, merging and detachment and their effects on the boiling heat transfer characteristics, which is difficult to do in the experiment studies. Therefore, in the present study, the boiling heat transfer process of structured surface is qualitatively analyzed by using LBM method.

Previous studies have shown that micro-nano structured surfaces can significantly enhance BHT performance, and the geometric size, shape and spacing between structures have significant effects on BHT characteristics and bubble dynamics behavior in the boiling process. However, most of the numerical simulation studies on the influence factors of the geometric shape of the structured surface were to design various groove shapes on the structured surface to form stable nucleation sites, or to design the structural surface with a series of micro-pillars. There are few studies on the influence of raised structural surfaces of other shapes (such as triangular-raised structures formed by inclined heating surfaces), the mechanism and the effects of the spacing between the geometric structures on BHT characteristics and bubble dynamics behavior have not been fully understood. Therefore, the pool boiling process and the enhanced heat transfer characteristics of such structured surfaces are studied in the current study. A pseudopotential multiphase LB model coupled FDM proposed by Li et al. (2015) was adopted in the simulations. The model had been verified and employed successfully in several subsequent studies of pool boiling (Li et al. 2015, 2018). In the present work, the effects of the microstructure geometric shapes and the structure spacing on the bubble dynamic behavior and heat transfer characteristics are studied in detail, the phenomena during the boiling process are analyzed and the mechanism of heat transfer enhancement is revealed. The nucleation, growth, coalescence, detachment and rising of bubbles during the boiling process on the structured surface are presented. The distribution of heat flux with time and space on different structured surfaces are obtained and analyzed in detail. The current study can take a step foward the obtantion of a better revealing and understanding of the physical mechanisms behind the enhancement of heat transfer efficiency due to structured surfaces and the interaction between bubble dynamics and microstructure and its effect on boiling heat transfer characteristics. 


\section{NUMERICAL MODEL}

In the current study, the pseudopotential multiphase flow LB model is applied to solve the gas-liquid twophase flow problem in the boiling process, the twophase density, the evolution of the interface, and the velocity variation within the flow field are obtained by solving the LB equation describing the density distribution function. The LB equation describing the density distribution function with multiple relaxation collision operators can be expressed as (Li et al. 2013)

$$
\begin{aligned}
& f_{i}\left(\boldsymbol{x}+\boldsymbol{e}_{i} \delta_{t}, t+\delta_{t}\right)=f_{i}(\boldsymbol{x}, t)- \\
& \left(\boldsymbol{M}^{-1} \boldsymbol{S} \boldsymbol{M}\right)_{i j}\left(f_{j}-f_{j}^{e q}\right)_{(\boldsymbol{x}, t)}+\delta_{t} F_{i}^{*}(\boldsymbol{x}, t)
\end{aligned}
$$

where $f_{i}$ and $f_{i}^{\text {eq }}$ are the function of density and density equilibrium distribution, respectively, $x$ is the spatial position, $\delta_{t}$ is the time step, $\boldsymbol{M}$ is a transformation matrix with orthogonal property, $\boldsymbol{e}_{i}$ is the discrete velocity in the $i$ direction, $F_{i}{ }^{*}$ is the forcing term within the velocity space, $\boldsymbol{S}$ is a diagonal matrix containing relaxation time, which is given by

$$
S=\operatorname{diag}\left(\tau_{0}^{-1}, \tau_{1}^{-1}, \tau_{2}^{-1}, \tau_{3}^{-1}, \tau_{4}^{-1}, \tau_{5}^{-1}, \tau_{6}^{-1}, \tau_{7}^{-1}, \tau_{8}^{-1}\right)
$$

The relaxation times are given by $\tau_{0}=\tau_{3}=\tau_{5}=1, \tau_{1}=$ $\tau_{2}=1.25, \tau_{4}=\tau_{6}=1 / 1.1$ in the current study. The shear viscosity determines the relaxation time $\tau_{7}$ and $\tau_{8}\left(\tau_{7}\right.$ $=\tau_{8}$ ). Multiplying the orthogonal matrix $\boldsymbol{M}$, the right expression of equation 1 can be transformed into

$$
\boldsymbol{m}^{*}=\boldsymbol{m}-\boldsymbol{S}\left(\boldsymbol{m}-\boldsymbol{m}^{e q}\right)+\delta_{t}(\boldsymbol{I}-0.5 \boldsymbol{S}) \boldsymbol{O}
$$

where $\boldsymbol{m}=\boldsymbol{M} \boldsymbol{f}, \boldsymbol{m}^{\mathrm{eq}}=\boldsymbol{M} \boldsymbol{f}^{\mathrm{eq}},(\boldsymbol{I}-0.5 \boldsymbol{S}) \boldsymbol{O}=\boldsymbol{M} \boldsymbol{F}^{*}, \boldsymbol{I}$ and $\boldsymbol{O}$ are the unit tensor and the forcing term within the moment space, respectively. The equilibria $\boldsymbol{m}^{\text {eq }}$ can be written as follows:

$$
\begin{aligned}
& \boldsymbol{m}^{e q}=\rho\left(1,-2+3|\boldsymbol{u}|^{2}, 1-3|\boldsymbol{u}|^{2}, u_{x},-u_{x},\right. \\
& \left.u_{y},-u_{y}, u_{x}^{2}-u_{y}^{2}, u_{x} u_{y}\right)^{\mathrm{T}}
\end{aligned}
$$

where $\rho$ and $\boldsymbol{u}$ are the macroscopic density and velocity, respectively. The macroscopic density and velocity can be obtained as follows:

$$
\rho=\sum_{i} f_{i}, \rho \boldsymbol{u}=\sum_{i} \boldsymbol{e}_{i} f_{i}+0.5 \delta_{t} \boldsymbol{F}
$$

where $\boldsymbol{F}$ given by $\boldsymbol{F}=\boldsymbol{F}_{m}+\boldsymbol{F}_{b}+\boldsymbol{F}_{f}$ is the total force in the current study. $\boldsymbol{F}_{m}$ and $\boldsymbol{F}_{b}$ are the fluid-fluid and fluid-solid pseudopotential interaction force, respectively. $\quad \boldsymbol{F}_{f}$ is the buoyancy force. The interaction force $\boldsymbol{F}_{m}$ is given by

$$
\boldsymbol{F}_{m}=-G \psi(\boldsymbol{x}) \sum_{i} w_{i} \psi\left(\boldsymbol{x}+\boldsymbol{e}_{i}\right) \boldsymbol{e}_{i}
$$

where $G$ is the strength of fluid-fluid interaction, $w_{i}$ and $\psi$ are the weights and the pseudopotential, respectively. For the D2Q9 lattice, the weights are given as $w_{1-4}=1 / 3, w_{5-8}=1 / 12$. The pseudopotential is given by (Yuan and Schaefer 2006)
$\psi(x)=\sqrt{\frac{2\left(p_{E O S}-\rho c_{s}^{2}\right)}{G c^{2}}}$

where $c_{\mathrm{s}}$ is the lattice speed in D2Q9 model, $c=1$ is the lattice constant, and $P_{E O S}$ is the non-ideal state equation. In the current study, the state equation of Peng-Robinson(P-R) is used (Yuan and Schaefer 2006)

$p_{E O S}=\frac{\rho R T}{1-b \rho}-\frac{a \varphi(T) \rho^{2}}{1+2 b \rho-b^{2} \rho^{2}}$

$\psi(T)=\left[1+\left(0.37464+1.54226 \kappa-0.26992 \kappa^{2}\right)\left(1-\sqrt{T / T_{\mathrm{c}}}\right)\right]^{2}$

$a=0.45724 R^{2} T_{c}^{2} / p_{c}, b=0.0778 R T_{c} / p_{c}$

where $\kappa$ is the acentric factor given by $\kappa=0.344$ (Yuan and Schaefer 2006), $T_{c}$ is the critical temperature. In the current work, the parameter $R, b$, $a$, are given by $R=1, b=2 / 21$ and $a=3 / 49$ (Li et al. 2015)

In order to achieve the wetting of solid wall and the adjustment of contact angle, in addition to the $F_{m}$, the fluid-solid pseudopotential interaction force $F_{\mathrm{b}}$ is also important, which is taken as

$\boldsymbol{F}_{b}=-G_{w} \psi(x) \sum_{i} \omega_{i} \psi(x) s\left(\boldsymbol{x}+\boldsymbol{e}_{i}\right) \boldsymbol{e}_{i}$

where $s\left(x+e_{i}\right)=1.0$ is for solid phase, while $s\left(x+e_{i}\right)$ $=0$ is for fluid phase, $G_{\mathrm{w}}$ is a parameter that can align the interaction force, and $\omega_{i}$ are the weights, which are described as $\omega_{i}=w_{i} / 3$. The buoyant force $F_{f}$ is given by

$$
F_{f}=\left(\rho-\rho_{\text {ave }}\right) \boldsymbol{g}
$$

where $\boldsymbol{g}$ and $\rho_{\text {ave }}$ are the gravitational acceleration and the average density among the computational domain, respectively. In the current study, an improved forcing scheme ( $\mathrm{Li}$ et al. 2013) is used for the forcing term $\boldsymbol{O}$ to achieve thermodynamic consistency. $\boldsymbol{O}$ is given by

$$
\begin{aligned}
& \boldsymbol{O}=\left[0,6 \boldsymbol{u} \cdot \boldsymbol{F}+\frac{\sigma\left|\boldsymbol{F}_{m}\right|^{2}}{\psi^{2} \delta_{t}\left(\tau_{1}-0.5\right)},-6 \boldsymbol{u} \cdot \boldsymbol{F}-\frac{\sigma\left|\boldsymbol{F}_{m}\right|^{2}}{\psi^{2} \delta_{t}\left(\tau_{2}-0.5\right)},\right. \\
& \left.F_{x},-F_{x}, F_{y},-F_{y}, 2\left(u_{x} F_{x}-u_{y} F_{y}\right), u_{x} F_{y}+u_{y} F_{x}\right]^{\mathrm{T}}
\end{aligned}
$$

where parameter $\sigma$ that can tune the condition of the mechanical stability is set to 1.2 ( $\mathrm{Li}$ et al. 2013) for achieving the consistency of the thermodynamic.

For the solution of temperature field in the present study, the viscous heat dissipation is neglected and the target equation is taken as (Li et al. 2018)

$$
\begin{aligned}
& \rho c_{v}\left(\partial_{t} T+\boldsymbol{u} \cdot \nabla T\right)=\nabla \cdot(\lambda \nabla T)- \\
& T\left(\frac{\partial p_{E O S}}{\partial T}\right)_{\rho} \nabla \cdot \boldsymbol{u}
\end{aligned}
$$


where $c_{\mathrm{v}}$ and $\lambda$ are the specific heat at constant volume and the thermal conductivity, respectively. The Eq. (14) can be transformed into

$$
\begin{aligned}
& \partial_{t} T=-\boldsymbol{u} \cdot \nabla T+\frac{1}{\rho c_{v}} \nabla \cdot(\lambda \nabla T)- \\
& \frac{T}{\rho c_{v}}\left(\frac{\partial p_{E O S}}{\partial T}\right)_{\rho} \nabla \cdot \boldsymbol{u}
\end{aligned}
$$

The Eq. (15) is solved by using FDM. The right expression of Eq. (15) is denoted by $K(T)$. The Eq. (15) is discretized in time and space. The isotropic central difference schemes (Lee and Lin 2005) are used to evaluate the spatial gradient and the Laplacian for the spatial discretization. The classical fourth-order Runge-Kutta scheme (Liu et al. 2013) is used for time discretization.

$T^{t+\delta_{t}}=T^{t}+\frac{\delta_{t}}{6}\left(h_{1}+2 h_{2}+2 h_{3}+h_{4}\right)$

where $h_{1}, h_{2}, h_{3}$, and $h_{4}$ are given as follows:

$h_{1}=K\left(T^{t}\right)$

$h_{2}=K\left(T^{t}+0.5 \delta_{t} h_{1}\right)$

$h_{3}=K\left(T^{t}+0.5 \delta_{t} h_{2}\right)$

$h_{4}=K\left(T^{t}+\delta_{t} h_{3}\right)$

\section{NUMERICAL RESULTS AND DISCUSSION}

\subsection{The Simulation Setup}

Numerical simulations are conducted in the rectangular cavity with the calculation area of $L x \times L y$ $=450$ 1.u. $\times 200$ 1.u. (1.u. represents lattice units). As shown in Fig. 1, the top and bottom of the cavity are solid walls, and the bottom wall is textured with microstructures. Figure 2 shows the different types of applicable geometry (Schubert et al. 2011) of the structured surface. In the present study, three structured surfaces that are close to the actual manufacturing conditions and similar to the applicable geometry of the structured surface are designed for comparison and analysis, which are defined as structure 1(square structures), structure 2(triangular serrated structures) and structure 3 (triangular-raised structures). The fixed width and height of the microstructure are set to 40 1.u. and 20 1.u., respectively. The spacing between the two adjacent microstructures is selected as $d=110$ l.u. The bottom of the cavity and the surface of the microstructures are the heating surface. The heating surface is set as the constant temperature boundary. It should be mentioned in the current study that the conjugate heat transfer is not considered. Although there is a difference in the simulation of boiling heat transfer between using constant temperature boundary conditions and considering conjugate heat transfer, according to the conclusion drawn by (Zhao et al. 2019c): in the nucleate boiling stage, the difference between considering and not considering conjugate heat transfer is small, and the main difference lies in transitional boiling. The current study is mainly concerned with the heat transfer characteristics in the nucleate boiling stage, thus, the numerical difference caused by the constant temperature boundary condition in the simulation of nucleate boiling is small. And although the constant temperature boundary condition is different from the actual condition, it can also be used to qualitatively analyze the bubble dynamics and boiling heat transfer characteristics in the boiling simulation. For example, Li obtained boiling curves (Li et al. 2015) and heat transfer characteristics under complex structures (Li et al. 2018) by using constant temperature boundary conditions in the simulation of boiling heat transfer.

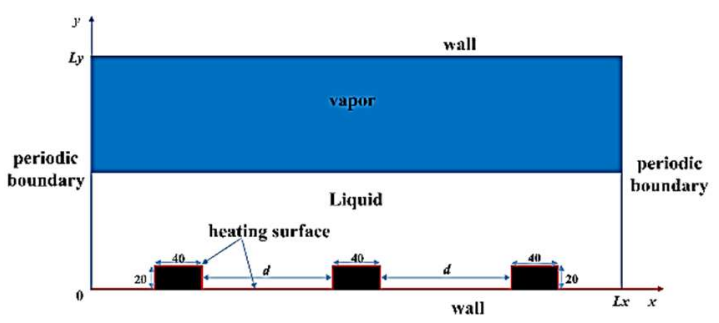

(a)

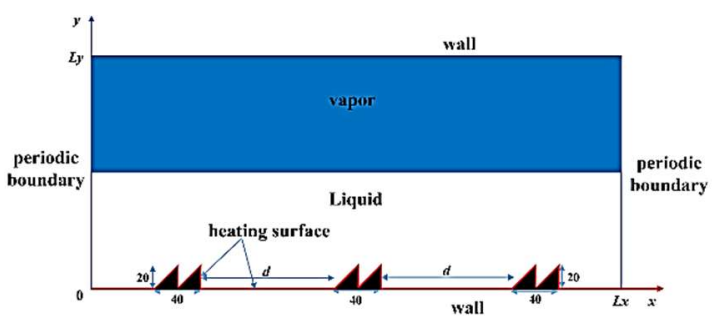

(b)

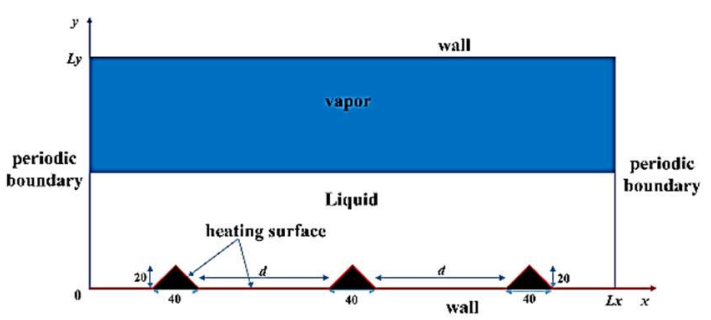

(c)

Fig. 1. Schematic diagram of structure 1(a), structure 2(b) and structure 3(c).

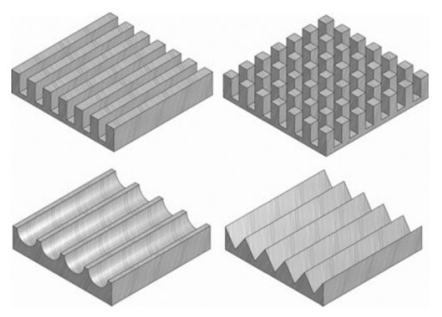

Fig. 2. Different types of applicable geometry of the structured surface.

The hydrophilic is set as the wettability of the heating surface. At the initial time, the vapor-liquid region is 
divided, and the calculation area is set as liquid $(0 \leq$ $\mathrm{y} \leq 0.5 \mathrm{Ly}$ ), the remaining area is set as vapor. The temperature is set at the coexistence temperature $T_{\mathrm{s}}$ $=0.86 T_{\mathrm{c}}$, at which the corresponding vapor-liquid coexistence densities are $\rho_{L} \approx 6.5$ and $\rho_{V} \approx 0.38$. The temperature on the heating surface is taken as $T_{b}$, while the top boundary temperature remains at $T_{s}$. The superheat on the heating surface is given by $\Delta T$ $=T_{b}-T_{s}$. The $J a$ number is introduced to evaluate the degree of superheat of the heating surface, which is given by:

$$
J a=c_{v}\left(T_{b}-T_{s}\right) / h_{l v}
$$

where $c_{v}$ is the specific heat at constant volume, which is set to 6.0 , the $h_{l v}$ is the specific latent heat of fluid, which is determined to be 0.58 according to the literature (Gong and Cheng 2013). The formula for calculating thermal conductivity is given by $\lambda=$ $\rho c_{\nu} \chi$, which is proportional to the density with $c_{\nu} \chi=$ 0.07 , where $\chi$ is the thermal diffusion coefficient. The kinematic viscosity of the liquid is set to $v_{L}=0.1$ and the vapor is taken as $v_{V}=0.5 / 3$, and the acceleration of gravity is chosen as $g=0.00003$. For the wettability of the heating surface, the $G_{\mathrm{w}}$ is set to $G_{\mathrm{W}}$ $=0.065$, which gives the equilibrium contact angle $\theta$ around $50^{\circ}$ (defined in the liquid phase ) at the saturation temperature. During the first 1000 timesteps, the simulations are carried out without the temperature solver. The units of all physical quantities involved in the current study are lattice units except for the dimensionless $J a$ number. It can be found from (He et al. 2009) that lattice units and physical units can be converted into each other.

\subsection{The Boundary Settings}

In the process of simulation, Zou-He non-slip boundary scheme (Zou and He 1997) is employed to the top and bottom walls: the realization of the nonslip scheme of the wall boundary lies in solving for the unknown distribution functions on the boundary. In order to achieve the non-slip boundary scheme for the wall, the velocity at the wall is set to 0 , the formula $\rho \boldsymbol{u}=\sum_{i} f_{i} \boldsymbol{e}_{i}+0.5 \delta_{t} \boldsymbol{F}$ and $f_{i}-f_{i}^{e q}=f_{i^{\prime}}-f_{i^{\prime}}^{e q}$ ( $i$ 'is the opposite direction of $i$ ) are used to solve for the unknown distribution functions on the wall. The periodic boundary condition is applied to the left and right boundaries. The constant temperature boundary is applied to the top and bottom walls. Since the inclination of structures 2 and 3 is $45^{\circ}$, the surface of structures 2 and 3 can be directly discretized to the lattice points without complicated interpolation calculations, as shown in Fig. 3. The unknown distribution functions are solved for these lattice points, which are solved by associating the two above equations and the velocity values on the boundary (the velocity is set to 0 ) to achieve the nonslip boundary scheme for the inclined boundaries.

\subsection{Model Verification}

\subsubsection{D2 Law Verification}

In LBM simulation of vapor-liquid phase transition, the $\mathrm{D}^{2}$ law for describing droplet evaporation is utilized to verify the phase change model in the

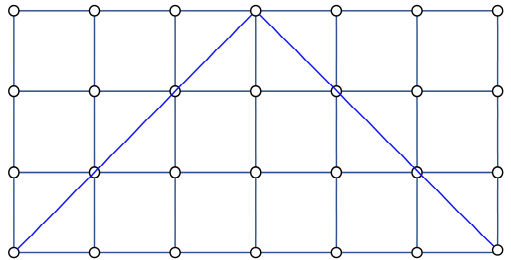

Fig. 3. Mesh discretization for inclined walls.

present study. In the initial setup, the entire computational domain is set to $2001 . u$. $\times 200$ l.u., in which a droplet with a diameter of 60 1.u. is suspended in the central area of the calculation domain, while the rest of the domain is filled with vapor. The initial temperature in the area where the droplet is located is set to $T_{s}=0.86 T_{c}$, and the temperature in the remaining area is set to $T_{c}$, thus, the $J a$ number is equal to 0.158 . The periodic boundary conditions and constant temperature boundary conditions $T=T_{c}$ are employed at the boundary. In the simulation of droplet evaporation, viscous heat dissipation is neglected and there is no buoyancy force. The $c_{v}$ and $\lambda$ are constant. The constant $c_{v}$ is taken as 6.0 and the kinematic viscosity is chosen as 0.1. As shown in Fig. 4, the square of droplet diameter varies linearly with time, which conforms to the $\mathrm{D}^{2}$ law of droplet evaporation. Therefore, the model utilized in the current study is reliable in simulating vapor-liquid phase change.

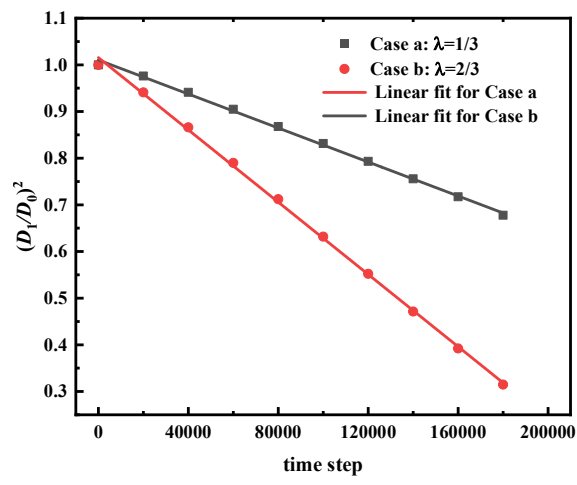

Fig. 4. D2 law verification.

\subsubsection{Simulation of Bubble Nucleation and Departure}

In the process of nucleate boiling, the bubble nucleation and departure with high temperature at three grids in the bottom center wall is simulated. In the initial setting of the simulation, the grid size of $L x \times L y=150 \quad 1 . u . \times 300$ l.u. is used in the computational domain. The calculation region of $0 \leq$ $\mathrm{y} \leq 0.5 L y$ is liquid, and the remaining domain is vapor. The kinematic viscosity is taken as 0.1 , The constant $c_{v}$ is taken as 5.0, and the thermal diffusivity is chosen as 0.06. Except that the temperature of the three center grids is fixed to high temperature $T_{h}=$ $1.25 T_{c}$, the temperature of the bottom wall is fixed to $T_{s}=0.86 T_{c}$, and the initial temperature of the rest of the domain is taken as $T_{s}$, thus, the $J a$ number is 
equal to 0.44 . The equilibrium contact angle is taken as $\theta \approx 45^{\circ}$. The periodic boundary conditions are employed at the left and right sides of the computational domain. As shown in Fig. 5, the bubble departure diameter obtained in the current study is proportional to $g^{-0.5}$, which satisfies the relationship obtained in the literature (Friz 1935). Therefore, it fully shows that the phase change model adopted in the present study has excellent reliability in simulating boiling.

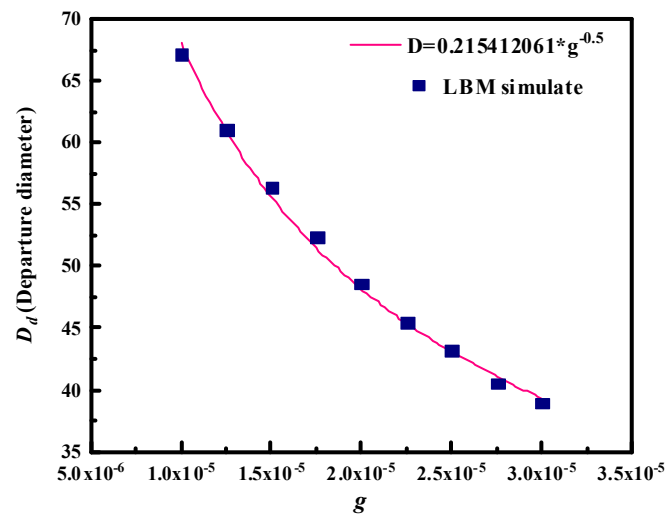

Fig. 5. The effect of gravity on bubble departure diameter.

\subsection{The Effects of Microstructure Geometry on Bubble Dynamics Behavior and BHT Characteristics}

In the current study, three different kinds of structured surfaces are used to study the effects of microstructure geometry on bubble dynamic and heat transfer characteristics, namely structure 1, structure 2, structure 3. Figures 6 and 7 show a comparison of the average heat flux and heat transfer coefficient on the surface of structures 1, 2 and 3 during nucleate boiling, respectively. Each point in Fig. 6. represents the average heat flux of the heating surface at such $J a$ number, which is the time average of the transient heat flux during the time steps of 1000 to 40000 , and the transient heat flux is the spatial average of the local heat flux along the heating surface, and the local heat flux is calculated by Fourier heat conduction law. The heat transfer coefficient is defined as the average heat flux divided by superheat. According to the above definition, the average heat flux and heat transfer coefficient are calculated as:

$Q_{a v e}=\int_{1000}^{40000}\left(\int_{0}^{L_{w}}(-\lambda \partial T / \partial y) d x / L_{w}\right) d t / 39000$

$J=Q_{\text {ave }} / \Delta T$

where $Q_{a v e}$ is the average heat flux, $J$ is the heat transfer coefficient, $L_{w}$ is the total length of heating surface.

It can be seen from figures 6 and 7 that the average heat flux and heat transfer coefficient on the surface of structure 1 are higher than those of structures 2

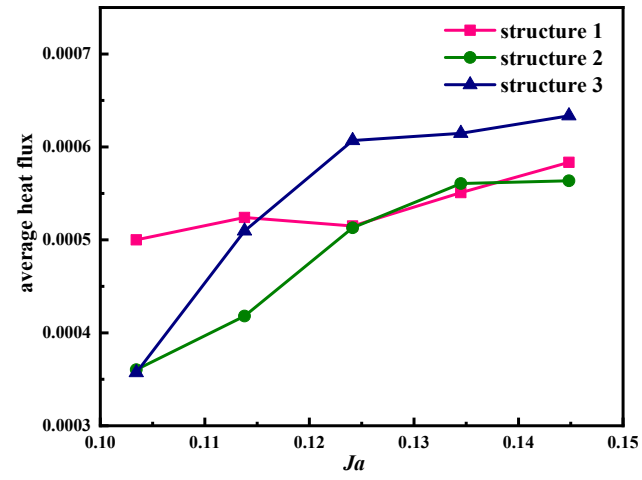

Fig. 6. Comparison of average heat fluxes on three kinds of structured surfaces under different $J a$ number.

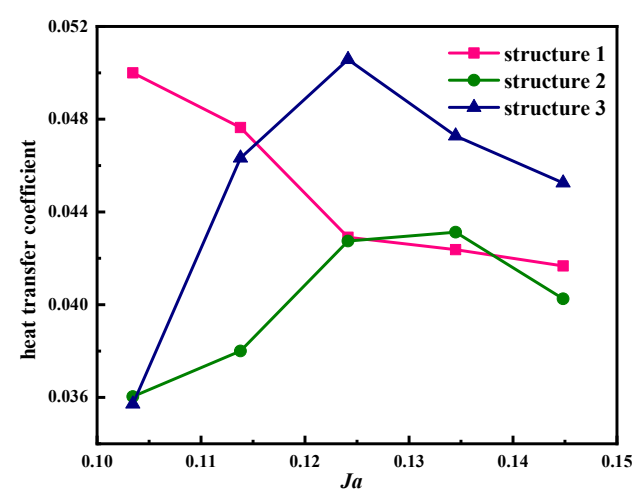

Fig. 7. Comparison of the heat transfer coefficient on three kinds of structured surfaces under different $J a$ number.

and 3 at the initial stage of nucleate boiling ( $\mathrm{Ja}=$ 0.103 and $J a=0.114$ ), thus, the performance of BHT is better. This is because the bottom corner of the columnar structure accelerates the nucleation of bubbles, and the top corner effect leads to the growth pattern of bubbles expanding, contracting, and pulling up on both sides of the structure, as shown in Fig. 8. This growth model leads to the enhancement of the disturbance to the flow field and the convection on the flat surface. Figures 6 and 7 also show that the average heat flux and heat transfer coefficient on the surface of structure 2 are almost smaller than those of structures 1 and 3. The reason is that in the nucleate boiling process, the nucleated and growing bubbles in the central groove of structure 2 do not break away from the wall with the separation of the merged bubbles, and remain in the grooves all the time, as shown in Fig. 9. Thus, this accelerates the process of bubble coalescence, and because the bubbles always cover the heating surface in the grooves, the BHT in the grooves deteriorates. Therefore, the BHT performance of structure 2 is almost weaker than that of structures 1 and 3 during nucleate boiling.

When the $J a$ number is $0.124-0.145$, nucleate boiling has been fully developed, Fig. 6 shows that the average heat flux on the structure 3 surface is larger than those of structures 1 and 2, and it is most evident 

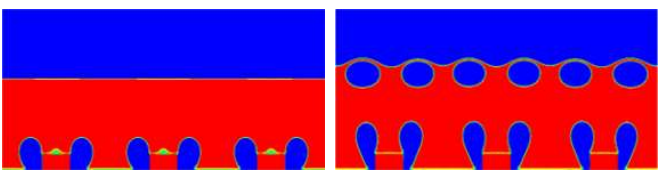

$J a=0.103, t=8000 \delta_{\mathrm{t}}$ $38000 \delta_{\mathrm{t}}$
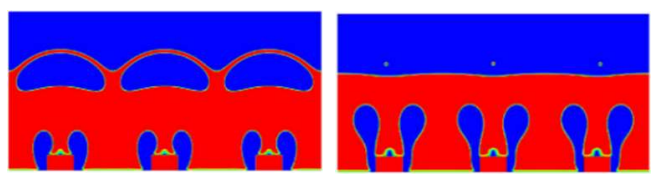

$J a=0.114, t=18300 \delta_{\mathrm{t}}$ $37900 \delta_{\mathrm{t}}$

$J a=0.114, t=$

Fig. 8. Snapshots of the process of boiling on structure 1 surface at $J a=0.103$ and $J a=0.114$.

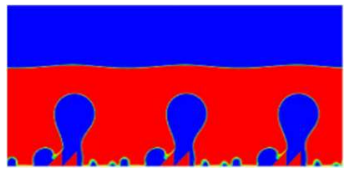

$J a=0.124, t=8200 \delta_{\mathrm{t}}$ $15000 \delta_{\mathrm{t}}$

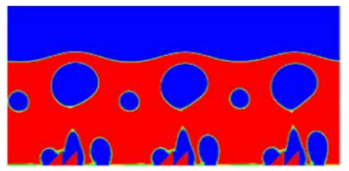

$J a=0.124, t=27100 \delta_{\mathrm{t}}$ $37300 \delta_{\mathrm{t}}$

Fig. 9. Snapshots of the process of boiling on structure 2 surface at $J a=0.124$.

when the $J a$ number is 0.124 . The heat transfer coefficient curve shows that when the $J a$ number is 0.124 , the BHT efficiency on the structure 3 surface is the highest among all three. Therefore, in the current study, we focus on the analysis of the boiling phenomenon and BHT characteristics on the structured surface when the $J a$ number is 0.124 . Fig. 10 shows the time evolution of transient heat fluxes on different structured surfaces. Compared with the boiling phenomenon between adjacent time-steps marked in the diagram, it can be found that the variation of heat flux at each time in the diagram is related to the motion of bubbles. Therefore, based on the motion of bubbles, the present study deeply explores the boiling heat transfer mechanism of three kinds of structured surfaces. From the boiling phenomenon in Fig. 11(a-c), it can be observed that for three kinds of structural surfaces, whenever bubbles expand on the heating surface and merge with each other during bubble nucleation and growth, these lead to the decline of the heat flux curve, resulting in the weakening of the BHT performance. On the other hand, whenever the growing and merged bubbles shrink, deform and detach on the heating wall, these lead to the upward movement of the heat flux curve, thus enhancing the BHT performance.
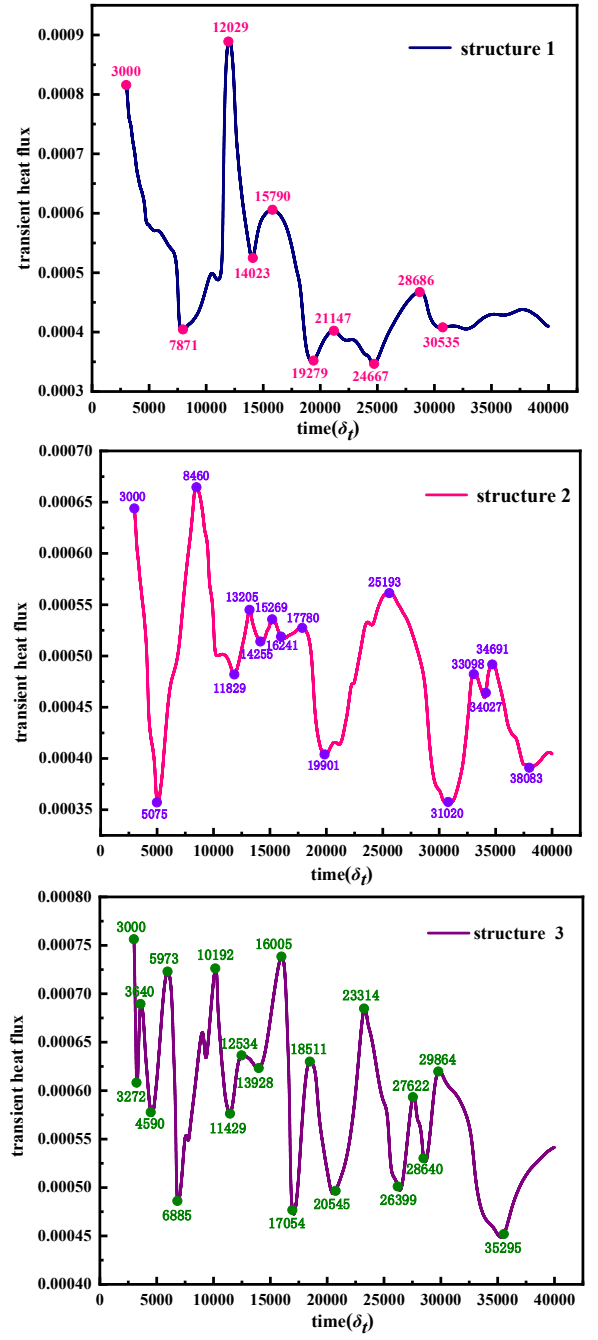

Fig. 10. Time evolution of transient heat fluxes on structures 1, 2 and 3 surfaces at $J a=0.124$.

During the boiling process in structures 2 and 3, a special bubble motion behavior is found to enhance BHT. Due to the inclined protruding structure in structures 2 and 3 and the flow field disturbance caused by the oscillation and deformation of the merged bubble in the process of gradually detaching from the wall, the continuous nucleation of small bubbles on the heating surface and the movement behavior of bubbles lateral migration are produced, as shown in Fig. 11(b, c). This process enhances the heat transfer performance. Therefore, combined with the above bubble dynamic behavior analysis, the bubble necking, deformation, detachment process and the movement behavior of bubbles lateral migration on the heating wall make the heating wall wetted again, thus enhancing the transient heat conduction of the superheated layer around the boiling site of the rewetting wall. At the same time, the growth, oscillation and detachment of bubbles also perturb the liquid and enhance the natural convection around the bubbles. This part of the enhanced convection around the bubble is called micro-convection. Therefore, in the present study, the main heat transfer mechanism of nucleate boiling on the three kinds of structured surface is the 
combined action of transient heat conduction and micro-convection. Because the bubbles located in the region between the two adjacent microstructures in structure 1 merge to form a large bubble after 15800 time steps, the vapor film remains on the flat surface all the time after the bubble detaches, as shown in Fig. 11(a). The BHT performance is weakened. On the other hand, the heat transfer performance of structure 2 is weakened because there are always bubbles in the central groove during the whole boiling process. The structure 3 has the best heat transfer performance due to its special structure, which accelerates the process of frequent necking and detachment of the merged bubbles from the wall and strengthens the lateral migration behavior of the bubbles, as shown in Fig. 11(c).
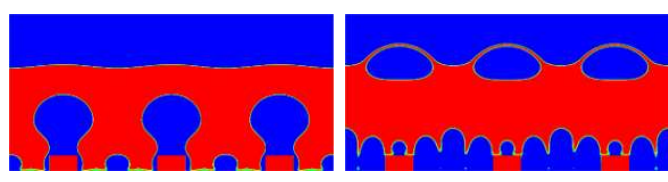

(a) $t=11300 \delta_{\mathrm{t}}$

(a) $t=18000 \delta_{\mathrm{t}}$
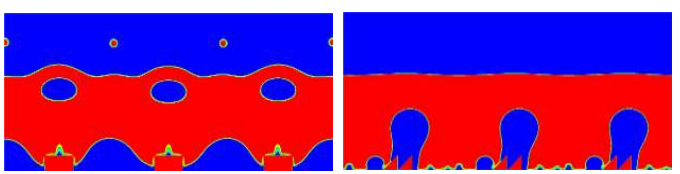

(a) $t=33000 \delta_{\mathrm{t}}$

(b) $t=7500 \delta_{\mathrm{t}}$
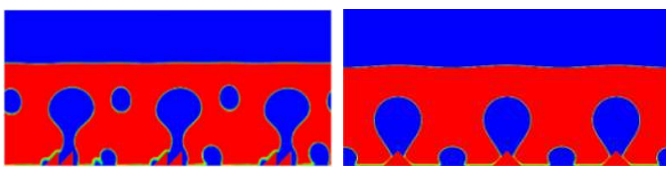

(b) $t=25000 \delta_{\mathrm{t}}$

(c) $t=9900 \delta_{\mathrm{t}}$
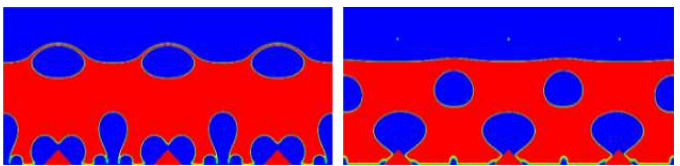

(c) $t=16600 \delta$

(c) $t=22400 \delta_{\mathrm{t}}$

Fig. 11. Snapshots of the process of boiling on structure 1(a), structure 2(b) and structure 3(c) surfaces at $J a=0.124$.

\subsection{The Effects of Spacing Between Microstructures on Bubbles Nucleation and BHT Characteristics}

Since that structure 3 shows better BHT performance in the process of nucleate boiling, and that the heat flux and heat transfer coefficient are higher than those of structures 1 and 2, structure 3 is used in the present study as the benchmark model to study the effects of the spacing between microstructures on bubble dynamics and BHT characteristics. In the current study, the effect of the spacing between microstructures on bubbles nucleation is discussed, and the motion behavior of bubbles and heat transfer characteristics with different spacing are analyzed emphatically when the $J a$ number is 0.124 . The distance between the microstructures is set to 0,22 ,
44, 66, 88, 110 1.u. Fig. 12 shows that the average heat flux varies with the distance between the microstructures when the $J a$ number is $0.124,0.134$, 0.145 . The average heat flux fluctuates with the increase of the distance between microstructures. Therefore, the variation of the spacing between microstructures has an important effect on the BHT performance of the heating surface.

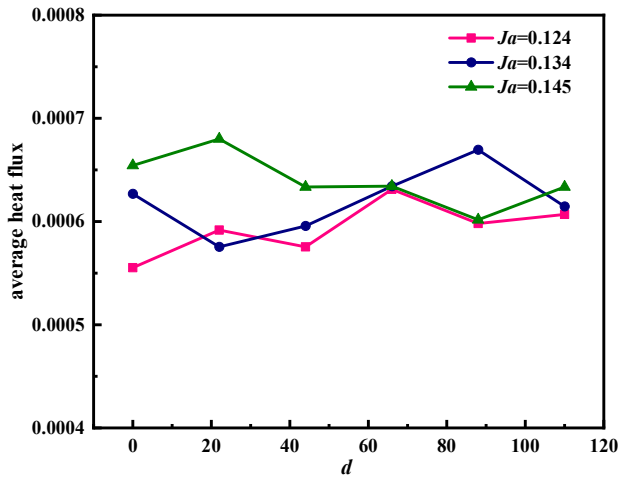

Fig. 12. Variation of the average heat fluxs with the distance between the microstructures at $J a=$ $0.124,0.134,0.145$.

Figure 13 depicts the distribution of time-averaged local heat flux on the heating surface when the $J a$ number is 0.124 and the spacing is $0,22,44,66,88$, 110 l.u., respectively. The time-averaged local heat flux is the time average of the local heat flux during the time steps of 1000 to 40000 . Figure 13 shows that the time-averaged local heat flux fluctuates on the heating surface, with multiple peaks and troughs. The trough represents the small time-averaged local heat flux in the region, which indicates that nucleating bubbles are often produced in the region. Therefore, it can be determined that this area is the main region of activation nucleation sites for producing bubbles. It can be seen from the Fig. 13 that the regions 1, 2 and 3 marked in the graph are in the trough of the curve with the time-averaged local heat flux being the smallest. Therefore, it can be explained that bubbles are often produced in these regions - the main nucleation region. By obtaining the coordinates of the points of the smallest timeaveraged local heat flux corresponding to the curve of each interval, it can be found that the coordinates exactly correspond to the intersection of the microstructure and the bottom wall. Therefore, the existence of microstructure accelerates the bubble nucleation, making the bottom corner the easiest and the fastest point to produce nucleating bubbles.

It can be seen from the Fig. 13 that when the distance between the microstructures increases from 0 to 22 , the region between the 1 and 2 in the figure does not produce the activated nucleation sites of bubbles. As the spacing increases to 44 , nucleation points begin to appear in the center of the region between the 1 and 2 , and when the spacing increases to 66 , two main bubble activation nucleation regions 6 and 7 develop. The activated nucleation sites of bubbles are mainly formed in these two regions. As the 

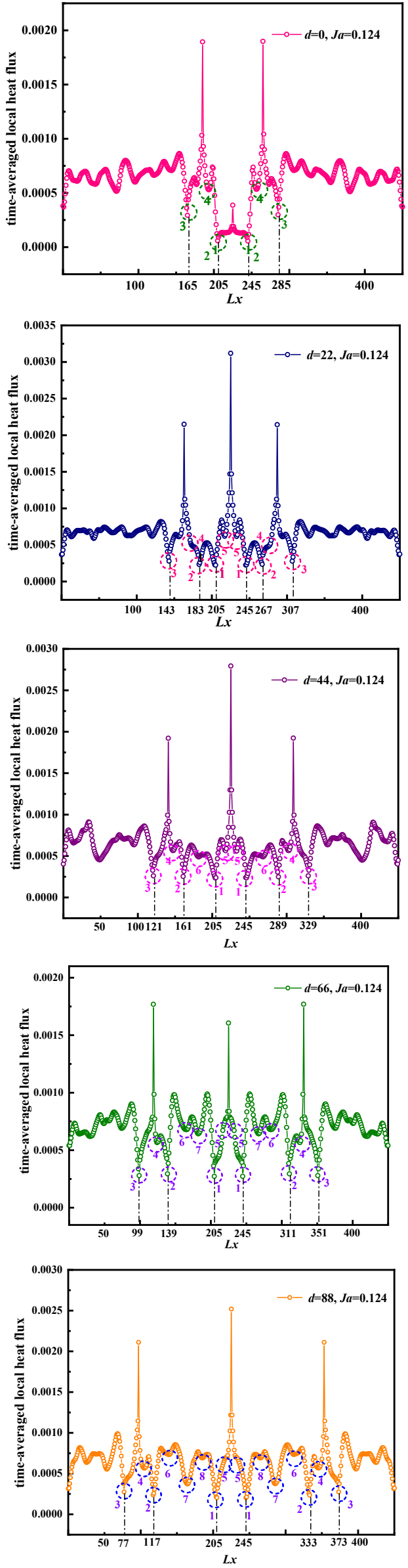

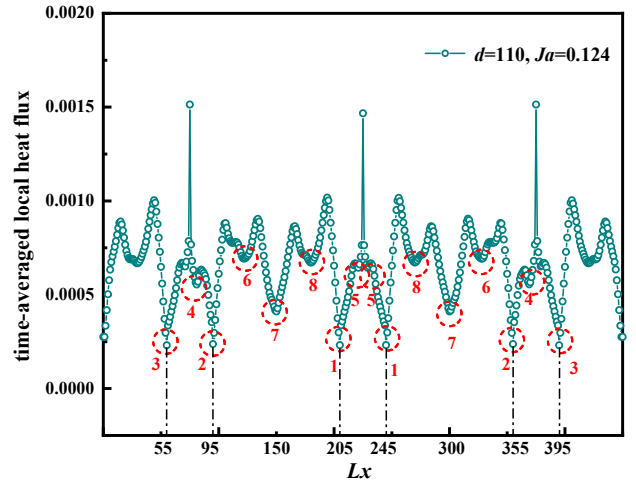

Fig. 13. Distribution of time-averaged local heat flux on the structure 3 surface at $J a=0.124$ and $\mathrm{d}=0,22,44,66,88,110$.

distance between microstructures continues to increase, the bubble activated nucleation region 8 is increased, and as the spacing increases to 110 , the 6 , 7 and 8-nucleation regions all develop, and they produce more activated nucleation sites, while the bubble 7-nucleation region in the center develop into the main bubble nucleation region with its bubble nucleation rate being significantly faster than that of the 6 and 8-regions. The spacing between the microstructures not only affects the generation of nucleation points in the the region between the 1 and 2 , but also has an important influence on the location of bubble activation nucleation on the surface of the microstructure, such as the 4,5-nucleation regions marked in the figure. Therefore, the variation of the distance between microstructures has an important effect on the generation of activated nucleation sites on the heating surface.

\subsection{The Effects of Spacing Between Microstructures on Bubble Dynamic Behavior}

In the current study, in addition to analyzing the effect of the spacing between microstructures on the nucleation of bubbles on the heating surface, the effect of the spacing between microstructures on the bubbles dynamic behavior is also discussed when the $J a$ number is 0.124 . When the distance between the microstructures is 0 , by observing the boiling process described by Fig. 14(a), the regions where the bubbles merge and detach are mainly on the three structured surfaces and the left and right boundaries. However, during the whole boiling process, the surface of the microstructure in the middle position is always covered with a vapor film, so this leads to the deterioration of heat transfer. Due to the disturbance of the flow field and the special microstructure of structure 3 , it can be observed that the nucleation bubbles near the surface of the microstructure migrate to the structured surface and the bubbles near the left or right boundary of the computational domain migrate to the left or right boundary, as shown in Fig. 14(a). As the spacing increases to 22, the boiling process described by Fig. 14(b) shows that the main merging region of the bubble changes, and the bubble merging and detaching no longer occurs on the structured surface 

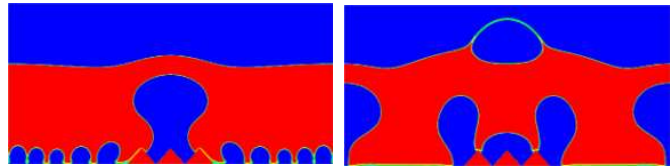

(a) $t=10000 \delta_{\mathrm{t}}$

(a) $t=17100 \delta_{\mathrm{t}}$
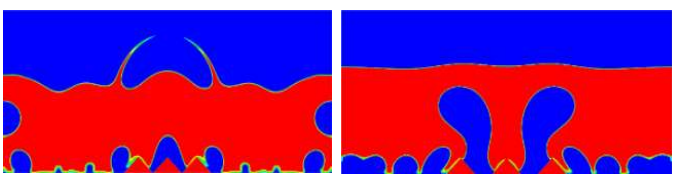

(a) $t=28300 \delta$

(b) $t=11300 \delta_{\mathrm{t}}$
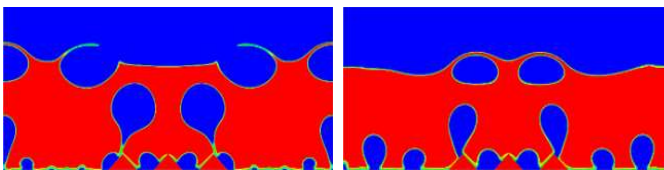

(b) $t=24600 \delta_{\mathrm{t}}$

(b) $t=37800 \delta_{\mathrm{t}}$
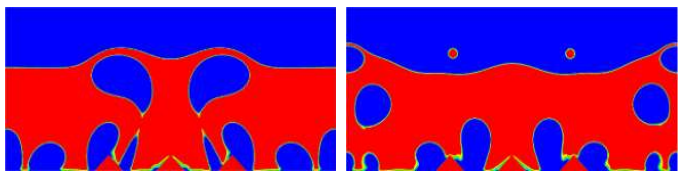

(c) $t=14800 \delta_{\mathrm{t}}$

(c) $t=25400 \delta_{\mathrm{t}}$
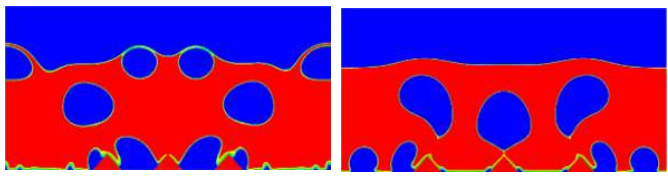

(c) $t=35800 \delta_{\mathrm{t}}$

(d) $t=11700 \delta_{\mathrm{t}}$
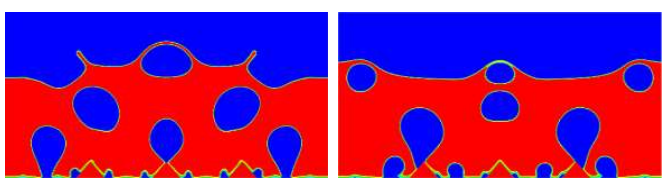

(d) $t=18000 \delta_{\mathrm{t}}$

(d) $t=25800 \delta_{\mathrm{t}}$
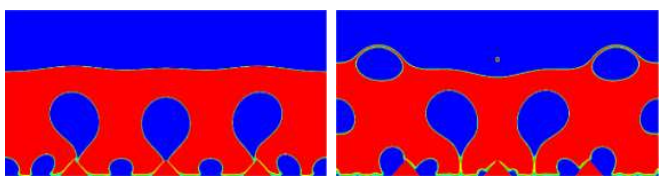

(e) $t=10500 \delta \mathrm{t}$

(e) $t=22900 \delta \mathrm{t}$
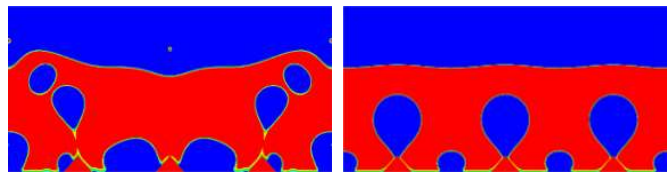

(e) $t=33400 \delta \mathrm{t}$

(f) $t=10200 \delta_{\mathrm{t}}$
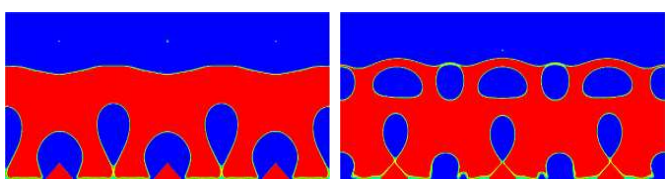

(f) $t=19600 \delta_{\mathrm{t}}$

(f) $t=28000 \delta_{\mathrm{t}}$

Fig. 14. Snapshots of the process of boiling on structure 3 surface at $J a=0.124$ and $d=0(a)$, 22(b), 44(c), 66(d), 88(e), 110(f).

of the middle region. The bubble coalescence and detachment mainly occur on the left and right structured surface and the left and right boundary, but the bubbles lateral migration phenomenon does not change. When the distance between the microstructures increases to 44, Fig. 14(c) shows that the main areas of bubble merging and detaching extend from the left and right sides of the structured surface to the flat surface between the microstructures, while the other ones remain unchanged. However, when the spacing continues to increase to 66 , not only the main areas of bubble merging and detachment have changed, but also the lateral migration direction of bubbles has changed. The main areas where bubbles merge and detach occur on all the structured surfaces, and the lateral migration direction of the bubbles nucleated on the flat surface moves toward the inclined surface of the microstructure, as shown in Fig. 14(d). As the distance between the microstructures continues to increase, Fig. 14(e, f) shows that the main regions of bubble merging and detaching occur not only on the surfaces of all microstructures, but also on the region center between the microstructures and on the left and right boundaries. The number of times of lateral migration of bubbles decreases with the increase of the spacing.

The bubbles dynamic behavior on the heating surface has an important effect on the BHT performance in nucleate boiling. As shown in Fig. 12, when the $\mathrm{Ja}$ number is 0.124 , the BHT performance is the best as the spacing increases to 66 , because the microstructure of structure 3 accelerates the frequent necking, deformation, and detachment of the merged bubbles compared with the flat surface, and strengthens the movement behavior of the bubbles lateral migration and the continuous nucleation process of the small bubbles on the heating surface. Such series of bubble dynamic behaviors enhance the transient heat conduction and micro-convection, with the strongest effect achieved when the distance between the microstructures is 66 , because when the spacing is 66, the main area of bubble merging and detaching is on the surface of the microstructure, the movement behavior of the bubbles lateral migration is frequent, and the lateral migration direction always moves to the inclined surface of the microstructure, as shown in Fig. 14(d). Therefore, bubble merging, necking, deformation and detachment on the structured surface accelerate, as well as the continuous nucleation of small bubbles on the heating surface.

\section{Conclusion}

In the current study, the pseudopotential multiphase LB model coupled FDM is used to simulate the pool boiling process and enhanced BHT characteristics on the structured surface. The effects of microstructure geometry and spacing on BHT characteristics and bubble dynamics behavior are studied in detail. Through our simulation study, the following main conclusions and findings are obtained.

The heat transfer performance of structure 1 is better than those of structures 2 and 3 at the initial stage of nucleate boiling ( $\mathrm{Ja}$ number is $0.103-0.114$ ) .The heat transfer performance of structure 2 is almost weaker than those of structures 1 and 3 during 
nucleate boiling. The boiling heat transfer performance of structure 3 is significantly better than those of structures 1 and 2 in the nucleate boiling ( $\mathrm{Ja}$ number is $0.124-0.145$ ).

On the three kinds of structured surfaces, when the bubbles expand on the heating surface and merge with each other during bubble nucleation and growth, the heat transfer performance is weakened. On the other hand, when the growing and merged bubbles shrink, deform and detach on the heating wall, the BHT performance is enhanced.

During the boiling process of structures 2 and 3, the continuous nucleation of small bubbles and the motion behavior of bubbles lateral migration caused by the oscillation and deformation of the merged bubbles are found. Such behaviors enhance the BHT performance. Most BHT is due to the re-wetting of the heating surface during bubble necking, deformation, detachment, and lateral migration, as well as enhanced convection around bubbles during bubble growth, oscillation and detachment, which indicates that the main BHT mechanism of nucleate boiling on the three kinds of structured surface is the combined action of transient heat conduction and micro-convection.

The variation of the distance between the microstructures has an important effect on the BHT performance of the heating surface. Moreover, the variation of the distance between the microstructures has an important influence on the generation of the activated nucleation sites on the heating surface. The existence of the microstructure accelerates the bubble nucleation, making the bottom corner the easiest and the fastest point to produce nucleating bubbles.

In the nucleate boiling stage, compared with the flat surface, the microstructure of structure 3 accelerates frequent necking, deformation and detachment of the merged bubbles from the heating wall, and strengthens the movement behavior of the bubbles lateral migration and the continuous nucleation of small bubbles on the heating surface. Therefore, structure 3 can enhance the transient heat conduction and micro-convection. The bubble dynamics behavior of the heating surface shows that the strengthening effect is the strongest as the spacing increases to 66 when the $J a$ number is 0.124 .

\section{ACKNOWLEDGEMENT}

Financial grants were received from the National Natural Science Foundation of China (11562011 and 51566012), Natural Science Foundation of Jiangxi Provence of China (20181BAB206031), De Montfort University through its distinguished ViceChancellor 2020 Programme, and the UK Science and Technology Facilities Council (STFC) through Batteries Early Career Researcher Award. Their support is greatly appreciated by the authors.

\section{REFERENCES}

Bi, J., D. M. Christopher, D. Zhao, J. Xu and Y.
Huang (2019). Numerical study of bubble growth and merger characteristics during nucleate boiling. Progress in Nuclear Energy 112, 7-19.

Chang, X., H. Huang, Y. P. Cheng and X. Y. Lu (2019). Lattice Boltzmann study of pool boiling heat transfer enhancement on structured surfaces. International Journal of Heat and Mass Transfer 139, 588-599.

Chu, K. H., R. Enright and E. N. Wang (2012). Structured surfaces for enhanced pool boiling heat transfer. Applied Physics Letters 100(24), 241603.

Dhir, V. K., G. R. Warrier and E. Aktinol (2013). Numerical simulation of pool boiling: a review. Journal of Heat Transfer 135(6).

Dong, B., Y. Zhang, X. Zhou, C. Chen and W. Li (2020). Numerical simulation of bubble dynamics in subcooled boiling along inclined structured surface. Journal of Thermophysics and Heat Transfer 35(1), 16-27.

Friz, W. (1935). Maximum volume of vapor bubbles. Physic. Zeitschz. 36, 379-354.

Gong, S. and P. Cheng (2013). Lattice Boltzmann simulation of periodic bubble nucleation, growth and departure from a heated surface in pool boiling. International Journal of Heat and Mass Transfer 64, 122-132.

Halon, T., B. Zajaczkowski, S. Michaie, R. Rulliere and J. Bonjour (2018). Enhanced tunneled surfaces for water pool boiling heat transfer under low pressure. International Journal of Heat and Mass Transfer 116, 93-103.

Hamzekhani, S., M. M. Falahieh, M. R. Kamalizadeh and M. Salmaninejad (2015). Bubble dynamics for nucleate pool boiling of water, ethanol and methanol pure liquids under the atmospheric pressure. Journal of Applied Fluid Mechanics 8(4), 893-898.

He, Y.L., Y. Wang and Q. Li (2009). Lattice Boltzmann method: theory and applications. Science, Beijing.

Jo, H., H. S. Ahn, S. Kang and M. H. Kim (2011). A study of nucleate boiling heat transfer on hydrophilic, hydrophobic and heterogeneous wetting surfaces. International Journal of Heat and Mass Transfer 54(25-26), 5643-5652.

Kim, D. E., D. I. Yu, D. W. Jerng, M. H. Kim and H. S. Ahn (2015a). Review of boiling heat transfer enhancement on micro/nanostructured surfaces. Experimental Thermal and Fluid Science 66, 173-196.

Kim, S. H., G. C. Lee, J. Y. Kang, K. Moriyama, M. H. Kim and H. S. Park (2015b). Boiling heat transfer and critical heat flux evaluation of the pool boiling on micro structured surface. International Journal of Heat and Mass Transfer 91, 1140-1147.

Lee, T. and C. L. Lin (2005). A stable discretization 
of the lattice Boltzmann equation for simulation of incompressible two-phase flows at high density ratio. Journal of Computational Physics 206(1), 16-47.

Li, Q., K. H. Luo and X. J. Li (2013). Lattice Boltzmann modeling of multiphase flows at large density ratio with an improved pseudopotential model. Physical Review E 87(5), 053301.

Li, Q., Q. J. Kang, M. M. Francois, Y. L. He and K. H. Luo (2015). Lattice Boltzmann modeling of boiling heat transfer: The boiling curve and the effects of wettability. International Journal of Heat and Mass Transfer 85, 787-796.

Li, Q., Y. Yu, P. Zhou and H. J. Yan (2018). Enhancement of boiling heat transfer using hydrophilic-hydrophobic mixed surfaces: a lattice Boltzmann study. Applied Thermal Engineering 132, 490-499.

Liu, H., A.J. Valocchi, Y. Zhang and Q. Kang (2013). Phase-field-based lattice Boltzmann finitedifference model for simulating thermocapillary flows. Physical Review E 87(1), 013010 .

O'hanley, H., C. Coyle, J. Buongiorno, T. Mckrell, L. W. Hu, M. Rubner and R. Cohen (2013). Separate effects of surface roughness, wettability, and porosity on the boiling critical heat flux. Applied Physics Letters 103(2), 024102.

Schubert, A., M. Hackert-Oschätzchen, G. Meichsner and M. Zinecker (2011). Design and realization of micro structured surfaces for thermodynamic applications. Microsystem technologies 17(9), 1471-1479.

Tang, Y., J. Zeng, S. Zhang, C. Chen and J. Chen (2016). Effect of structural parameters on pool boiling heat transfer for porous interconnected microchannel nets. International Journal of Heat and Mass Transfer 93, 906-917.

Thiagarajan, S. J., R. Yang, C. King and S. Narumanchi (2015). Bubble dynamics and nucleate pool boiling heat transfer on microporous copper surfaces. International Journal of Heat and Mass Transfer 89, 12971315.

Wei, J.J., L. J. Guo and H. Honda (2005). Experimental study of boiling phenomena and heat transfer performances of FC-72 over micro-pin-finned silicon chips. Heat and Mass Transfer 41(8), 744-755.

Xie, S., Q. Tong, Y. Guo, X. Li, H. Kong and J. Zhao (2020). The effects of surface orientation, heater size, wettability, and subcooling on the critical heat flux enhancement in pool boiling. International Journal of Heat and Mass Transfer 149, 119230.
Xie, S., M. Jiang, H. Kong, Q. Tong and J. Zhao (2021a). An experimental investigation on the pool boiling of multi-orientated hierarchical structured surfaces. International Journal of Heat and Mass Transfer 164, 120595.

Xie, S., X. Ma, H. Kong, S. Bai, M. Jiang and J. Zhao (2021b). The synergetic effects of the surface wettability and the patterned nanostructure on boiling heat transfer enhancement. International Journal of Heat and Mass Transfer 176, 121475.

Yuan, P. and L. Schaefer (2006). Equations of state in a lattice Boltzmann model. Physics of Fluids 18(4), 042101.

Zhang, Y., J. Bao, M. Yao, Y. Xie, Y. Huang and P. Li (2020). Non-orthogonal Multiple-RelaxationTime Lattice Boltzmann Simulation of Mixed Convection in Lid-Driven Porous Cavity with an Isothermally Heated Block. Journal of Applied Fluid Mechanics 13(5).

Zhao, W., J. Liang, M. Sun, X. Cai and P. Li (2019a). Hybrid phase-change lattice Boltzmann simulation of the bubble nucleation and different boiling regimes of conjugate boiling heat transfer. arXiv preprint arXiv:1911.10747.

Zhao, W., Y. Zhang and B. Xu (2019b). An improved pseudopotential multi-relaxation-time lattice Boltzmann model for binary droplet collision with large density ratio. Fluid Dynamics Research 51(2), 025510.

Zhao, W., J. Liang, M. Sun and Z. Wang (2021c). Investigation on the effect of convective outflow boundary condition on the bubbles growth, rising and breakup dynamics of nucleate boiling. International Journal of Thermal Sciences 167, 106877.

Zhao, W., Y. Zhang, W. Shang, Z. Wang, B. Xu and S. Jiang (2019). Simulation of droplet impacting a square solid obstacle in microchannel with different wettability by using high density ratio pseudopotential multiple-relaxation-time (MRT) lattice Boltzmann method (LBM). Canadian Journal of Physics 97(1), 93-113.

Zhou, P., W. Liu and Z. Liu (2019). Lattice Boltzmann simulation of nucleate boiling in micro-pillar structured surface. International Journal of Heat and Mass Transfer 131, 1-10.

Zonouzi, S. A., H. Safarzadeh, H. Aminfar and M. Mohammadpourfard (2018). Experimental study of subcooled boiling heat transfer of axial and swirling flows inside mini annular gaps. Journal of Applied Fluid Mechanics 11(1), 225232.

Zou, Q. and X. He (1997). On pressure and velocity boundary conditions for the lattice Boltzmann BGK model. Physics of Fluids 9(6), 1591-1598. 